VOL. $5(1971), 221-225$.

\title{
Remarks on theorems of Thompson and Freede
}

\author{
A.R. Amir-Moéz and C.R.Perry
}

Let $A$ be a hermitian transformation on an $n$-dimensional unitary space $E_{n}$, with proper values $a_{1} \geq \ldots \geq a_{n}$. Let $M$ be a proper subspace of $E_{n}$. Suppose $b_{1} \geq \ldots \geq b_{h}$ are the proper values of $A \mid M$ and $c_{1} \geq \ldots \geq c_{k}$ are the proper values of $A \mid M^{\perp}$. Let $i_{1}<\ldots<i_{p}$ and $j_{1}<\ldots<j_{r}$ be sequences of positive integers, with $i_{p} \leq k$ and $j_{p} \leq h$. Then

$$
\sum_{p=1}^{r} b_{i_{p}}+\sum_{p=1}^{r} c_{j_{p}} \leq \sum_{p=1}^{r} a_{p}+\sum_{p=1}^{r} a_{\left(i_{p}+j_{p}\right)} .
$$

This is a special case of one of the Thompson-Freede theorems which is proved by use of certain invariants.

Some very interesting generalizations of an inequality of Aronsajn have been given by Thompson and Freede [4]. In this note we give a sample of expressing these theorems in terms of linear transformations and give a proof using some invariants.

\section{Definitions and notations}

An $n$-dimensional unitary space will be indicated by $E_{n}$. The inner product of two vectors $\alpha$ and $\beta$ will be denoted by $(\alpha, \beta)$. An orthonormal set $\left\{\alpha_{1}, \ldots, \alpha_{k}\right\}$ will be indicated by $\left\{\alpha_{p}\right\}$ orthonormal. 
The subspace spanned by the set $\left\{\alpha_{1}, \ldots, \alpha_{k}\right\}$ will be denoted by $\left[\alpha_{1}, \ldots, \alpha_{k}\right]$. We write $\operatorname{dim} M=h$ if the dimension of the subspace $M$ is $h$.

If $A$ is a linear transformation on $E_{n}$ and if $M$ is a subspace of $E_{n}$, then we define a linear transformation $A \mid M$ as follows: if $\xi \in M$, let $[A \mid M] \xi=P A \xi$, where $P$ is the orthogonal projection on $M$. We observe that if $\alpha$ and $\beta \in M$, then

$$
([A \mid M] \alpha, \beta)=(P A \alpha, \beta)=(A \alpha, \beta) .
$$

It follows that if $A$ is hermitian, then $A \mid M$ is hermitian.

Given any sequence $i_{1} \leq \ldots \leq i_{k}$ of positive integers such that $i_{p} \geq p$, for $p=1, \ldots, k$, we define $\left(i_{1}^{\prime}, \ldots, i_{k}^{\prime}\right)$ recursively by $i_{k}^{\prime}=i_{k}$ and $i_{r}^{\prime}=\min \left(i_{r}, i_{r+1}^{\prime}-1\right)$, for $r=k-1, \ldots, 1,[1]$.

\section{Some preliminary theorems}

Let $H$ be a hermitian transformation on $E_{n}$ with proper values $m_{1} \geq \ldots \geq m_{n} \cdot$ Then

(1) $m_{1}+\ldots+m_{k}=\sup _{\left\{\xi_{i}\right\} \text { orthonormal }}\left[\left(H \xi_{I}, \xi_{1}\right)+\ldots+\left(H \xi_{k}, \xi_{k}\right)\right]$.

This theorem is due to Fan [2]. Further, if $i_{1} \leq \ldots \leq i_{k}$ is a sequence of positive integers such that $i_{p} \leq n$ and $i_{p} \geq p, p=1, \ldots, k$, then

(2)

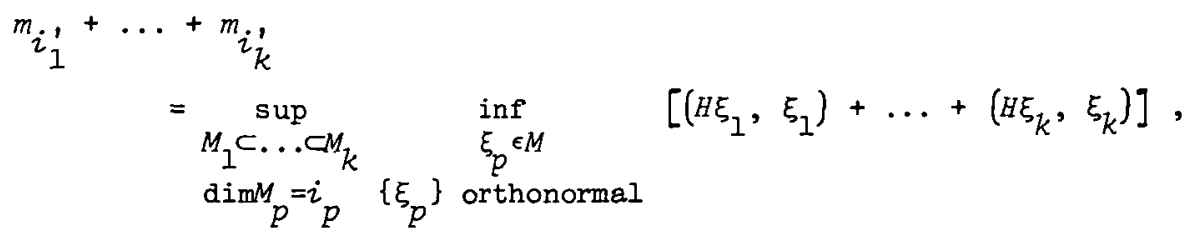

where $M_{p}$ is a subspace of $E_{n},[1]$.

3.

THEOREM. Let $A$ be a hermitian transformation on $E_{n}$ with proper 
values $a_{1} \geq \ldots \geq a_{n}$. Let $R_{1}, \ldots, R_{s}$ be proper subspaces of $E_{n}$ such that $E_{n}=R_{1} \oplus \ldots \oplus R_{s}$ and $R_{i}$ is orthogonal to $R_{j}$, for $i \neq j$. Let $\operatorname{dim} R_{q}=h_{q}, q=1, \ldots, s$. Suppose the proper values of $A \mid R_{q}$ are $b_{q 1} \geq \ldots \geq b_{q h}, q=1, \ldots, s$. Let $i_{q 1} \leq \ldots \leq i_{q r}$,

$q=1, \ldots, s$, be sequences of positive integers such that $i_{q p} \leq h_{q}$ and $i_{q p} \geq p$, for $p=1, \ldots, r$ and $q=1, \ldots, s$. Then

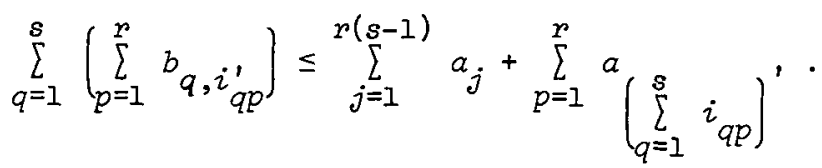

Proof. By $\S 2(2)$, there exist subspaces $M_{q 1} \subset \ldots \subset M_{q r} \subset R_{q}$, $q=1, \ldots, s$, with $\operatorname{dim} M_{q p}=i_{q p}$, for $p=1, \ldots, r$ and $q=1, \ldots, s$, such that

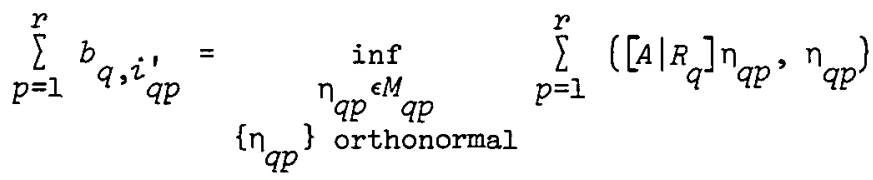

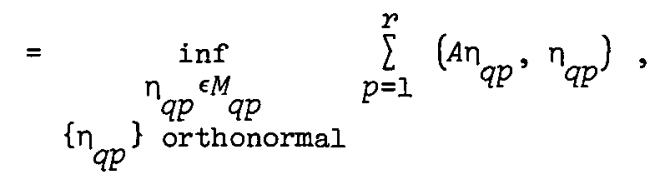

for $q=1, \ldots, s$.

Let $L_{p}=M_{1 p} \oplus \ldots \oplus M_{s p}, p=1, \ldots, r$. We observe that

$L_{1} \subset \ldots \subset L_{r} \subset E_{n}$ and $\operatorname{dim} L_{p}=\sum_{q=1}^{S} i_{q p}, p=1, \ldots, r$. Let

$\left\{\zeta_{1}, \ldots, \zeta_{p}\right\}$ be an orthonormal set in $E_{n}$ such that $\zeta_{p} \in L_{p}$, $p=1, \ldots, r$. Now, for each $p=1, \ldots, r$, it is clear that there exists an orthonormal set $\left\{n_{l p}, \ldots, n_{s p}\right\}$ such that $\zeta_{p} \in\left[n_{l p}, \ldots, n_{s p}\right]$ and $\eta_{q p} \in M_{q p}, q=1, \ldots, s$. Here the set

$\left\{n_{11}, \ldots, n_{1 r}, n_{2 I}, \ldots, n_{2 r}, \ldots, n_{s l}, \ldots, n_{s r}\right\}$ may not be linearly independent. But, it is clear, there exists an orthonormal set 
$\left\{n_{11}^{\prime}, \ldots, n_{1 r}^{\prime}, \ldots, n_{s l}^{\prime}, \ldots, n_{s r}^{\prime}\right\}$ such that

$\left[n_{11}, \ldots, n_{1 r}, \ldots, \eta_{s 1}, \ldots, n_{s r}\right] \subset\left[\eta_{11}^{\prime}, \ldots, n_{1 r}^{\prime}, \ldots, n_{s 1}^{\prime}, \ldots, n_{s r}^{\prime}\right]$ and $\eta_{q p}^{\prime} \in M_{q p}$, for $q=1, \ldots, s$ and $p=1, \ldots, r$. It is clear that $\left\{\zeta_{1}, \ldots, \zeta_{r}\right\}$ can be extended to an orthonormal set $\left\{\zeta_{1}, \ldots, \zeta_{s r}\right\}$ in such a way that $L=\left[n_{11}^{\prime}, \ldots, n_{1 r}^{\prime}, \ldots, \eta_{s 1}^{\prime}, \ldots, n_{s \gamma^{\prime}}^{\prime}\right]=\left[\zeta_{1}, \ldots, \zeta_{s \gamma}\right]$. Thus

$$
\sum_{q=1}^{s}\left(\sum_{p=1}^{r}\left(A n_{q p}^{\prime}, \eta_{q p}^{\prime}\right)\right)=\operatorname{trace}(A \mid L)=\sum_{i=1}^{s r}\left(A \zeta_{i}, \zeta_{i}\right) .
$$

By $\$ 2$ (1), it follows that

$$
\sum_{p=1}^{r(s-1)} a_{p} \geq \sum_{i=r+1}^{s r}\left(A \zeta_{i},, \zeta_{i}\right)
$$

Combining (3) and (4) we obtain

$$
\sum_{q=1}^{s}\left(\sum_{p=1}^{r}\left(A n_{q p}^{\prime}, n_{q p}^{\prime}\right)\right) \leq \sum_{i=1}^{r}\left(A \zeta_{i}, \zeta_{i}\right)+\sum_{j=1}^{r(s-1)} a_{j} .
$$

Using (2) and (5) we obtain

$$
\begin{aligned}
& \sum_{q=1}^{s}\left(\sum_{p=1}^{r} b_{q, i_{q p}^{\prime}}\right) \\
& \leq \sum_{j=1}^{r(s-1)} a_{j}+ \\
& K_{1} \subset \ldots \subset K_{r} \\
& \operatorname{dim} k_{p}=\sum_{q=1}^{s} i_{q p} \\
& \sum_{p=1}^{r}\left(A \delta_{p}, \delta_{p}\right) .
\end{aligned}
$$

Applying 52 (2) to the right side of (6) yields (1); thus the proof is complete.

\section{Remark}

We observe that the other results of Thompson and Freede can be proved in a manner similar to 53 . These results may also be obtained as corollaries to $\$ 3$ as was done in the Thompson and Freede paper. 


\section{References}

[1] Ali R. Amir-Moéz, "Extreme properties of eigenvalues of a Hermitian transformation and singular values of the sum and product of linear transformations", Duke Math. J. 23 (1956), 463-476.

[2] Ky Fan, "On a theorem of Weyl concerning eigenvalues of linear transformations. I", Proc. Nat. Acad. Sci. U.S.A. 35 (1949), $652-655$.

[3] H.L. Hamburger and M.E. Grimshaw, Linear transformations in $n$-dimensional vector spaces (Cambridge University Press, Cambridge, 1951).

[4] Robert C. Thompson and Linda J. Freede, "Eigenvalues of partitioned hermitian matrices", Bull. Austral. Math. Soc. 3 (1970), 23-37.

[5] Hermann Weyl, "Inequalities between the two kinds of eigenvalues of a linear transformation", Proc. Nat. Acad. Sci. U.S.A. 35 (1949), 408-411.

Texas Tech University,

Lubbock,

Texas, USA. 